\title{
Frontiers of Theoretical Physics
}


This page is intentionally left blank 
Frontiers of

Theoretical Physics

A General View of Theoretical Physics

at the Crossing of Centuries

Beijing, China $\quad$ 2-5 November 1999

\section{Editors}

\section{Fumihiko Sakata}

Ibaraki University, Japan

\section{Ke Wu \& En-Guang Zhao}

Academia Sinica, China 
Published by

World Scientific Publishing Co. Pte. Ltd.

P O Box 128, Farrer Road, Singapore 912805

USA office: Suite 1B, 1060 Main Street, River Edge, NJ 07661

UK office: 57 Shelton Street, Covent Garden, London WC2H 9HE

British Library Cataloguing-in-Publication Data

A catalogue record for this book is available from the British Library.

FRONTIERS OF THEORETICAL PHYSICS: A GENERAL VIEW OF THEORETICAL PHYSICS AT THE CROSSING OF CENTURIES

Copyright 12001 by World Scientific Publishing Co. Pte. Ltd.

All rights reserved. This book, or parts thereof, may not be reproduced in any form or by any means, electronic or mechanical, including photocopying, recording or any information storage and retrieval system now known or to be invented, without written permission from the Publisher.

For photocopying of material in this volume, please pay a copying fee through the Copyright Clearance Center, Inc., 222 Rosewood Drive, Danvers, MA 01923, USA. In this case permission to photocopy is not required from the publisher.

ISBN 981-02-4483-5

Printed in Singapore by Uto-Print 


\section{FOREWORD}

\section{YUAN-BEN DAI}

The "International Workshop on Frontiers of Theoretical Physics - a general view of theoretical physics at the crossing of centuries" was held on November 2-5, 1999 in Beijing. The conference was organized by the Institute of Theoretical Physics of Academia Sinica in China and the Faculty of Science of the Ibaraki University in Japan.

This is the first joint effort of the Institute of Theoretical Physics and the Ibaraki University to organize an academic conference. At the same time, this is also the first international conference held by the Institute of Theoretical Physics after the Academia Sinica embarked upon the "Creation of original knowledge project". The purpose of the conference was to provide a forum for the participants to exchange views and opinions on the development of theoretical physics in the new millennium.

There were a total of 31 participants mostly from many well known Japanese research institutions and universities. On the Chinese side, there were about 60 participants from the universities and research institutes in Beijing. A total of 35 talks were arranged during the conference. The fields include quantum mechanics, quantum field theory, superstrings, nuclear physics, condensed matter physics and etc. The speakers discussed and looked forward to the possible new developments in the new millennium.

During the conference the participants also took part in the ceremony in which the bronze bust of late professor Shoichi Sakata, a famous Japanese theoretical physicist and nice friend of the Chinese people, was unveiled at China Hall of Science and Technology. All participants took the opportunity to pay their hearty homage to professor S. Sakata.

The organizing committee is very grateful to the speakers and participants for their contributions to the success of the conference. Financial support from the National Natural Science Foundation of China, "PANDENG" Project of Theoretical Physics and Chinese Association of Science and Technology are also gratefully acknowledged. 
This page is intentionally left blank 


\section{世纪之交理论物理前沿问题国际研讨会 1999.11.2-5}

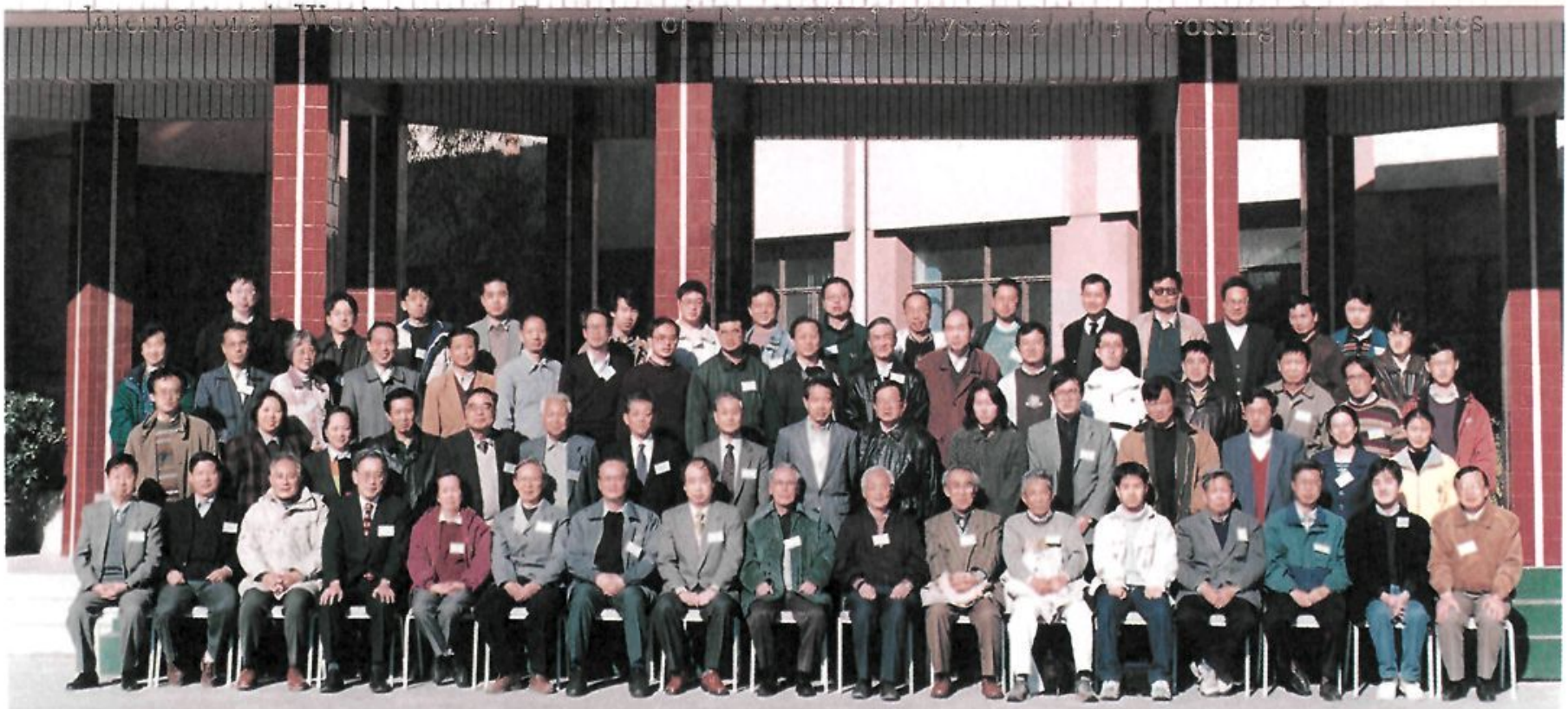

Participants in the International Workshop on Frontiers of Theoretical Physics: A General View of Theoretical Physics at the Crossing of Centuries. 
This page is intentionally left blank 


\section{List of Participants}

\begin{tabular}{|c|c|c|}
\hline 陈裕启 & Chen Yu-qi & Institute of Theoretical Physics, Academia Sinica \\
\hline 戴 柬 & Dai Jian & Physics Department, Beijing University \\
\hline 戴元本 & Dai Yuan-ben & $\begin{array}{l}\text { Institute of Theoretical Physics, Academia Sinica } \\
\text { dyb@itp.ac.cn }\end{array}$ \\
\hline 杜东生 & Du Dong-sheng & Institute of High Energy Physics, Academia Sinica \\
\hline 冯仁发 & Feng Ren-fa & $\begin{array}{l}\text { China Institute of Atomic Energy } \\
\text { rffeng@iris.ciae.ac.cn }\end{array}$ \\
\hline 藤田さほ & Saho Fujita & $\begin{array}{l}\text { Ibaraki University } \\
\text { nm9128@mcs.ibaraki.ac.jp }\end{array}$ \\
\hline 藤原滋泰 & Sigeyasu Fujiwara & $\begin{array}{l}\text { Ibaraki University } \\
\text { nm9129@mcs.ibaraki.ac.jp }\end{array}$ \\
\hline 藤原高德 & Takanori Fujiwara & $\begin{array}{l}\text { Ibaraki University } \\
\text { fujiwara@mito.ipc.ibaraki.ac.jp }\end{array}$ \\
\hline 高春媛 & Gao Chun-yuan & Techenical Physics Department, Beijing University \\
\hline 郭汉英 & Guo Han-ying & $\begin{array}{l}\text { Institute of Theoretical Physics, Academia Sinica } \\
\text { hyguo@itp.ac.cn }\end{array}$ \\
\hline 郭 华 & Guo Hua & Techenical Physics Department, Beijing University \\
\hline 苟 亮 & Guo Liang & Institute of High Energy Physics, Academia Sinica \\
\hline 郭 璐 & Guo Lu & $\begin{array}{l}\text { Institute of Theoretical Physics, Academia Sinica } \\
\text { guolu@itp.ac.cn }\end{array}$ \\
\hline 林 武美 & Takemi Hayashi & $\begin{array}{l}\text { Kogakkan University } \\
\text { hayashi@kogakkan-u.ac.jp }\end{array}$ \\
\hline 黄 梅 & Huang Mei & $\begin{array}{l}\text { Institute of High Energy Physics, Academia Sinica } \\
\text { mei@hp.ccast.ac.cn }\end{array}$ \\
\hline 黄 涛 & Huang Tao & Institute of High Energy Physics, Academia Sinica \\
\hline 石 橋 & Nobuyuki Ishibashi & $\begin{array}{l}\text { KEK } \\
\text { ishibash@post.kek.jp }\end{array}$ \\
\hline 神谷美子 & Yoshiko Kamiya & Nagoya University \\
\hline 菅野正吉 & Shokichi Kanno & $\begin{array}{l}\text { Ibaraki University } \\
\text { kanno@mito.ipc.ibaraki.ac.jp }\end{array}$ \\
\hline 小林 誠 & Makoto Kobayashi & $\begin{array}{l}\text { KEK } \\
\text { kobayath@kek.jp }\end{array}$ \\
\hline 仍宇平 & Kuang Yu-ping & Physics Department, Qsinghua University, Beijing \\
\hline 李重生 & Li Chong-sheng & Physics Department, Beijing University \\
\hline 厉光烈 & Li Guang-lie & Institute of High Energy Physics, Academia Sinica \\
\hline
\end{tabular}




\begin{tabular}{|c|c|c|}
\hline 李祝霞 & Li Zhu-xia & $\begin{array}{l}\text { China Institute of Atomic Energy } \\
\text { lizwux@ iris.ciae.ac.cn }\end{array}$ \\
\hline 廖 伟 & Liao Wei & Institute of Theoretical Physics, Academia Sinica \\
\hline 刘 纯 & Liu Chun & $\begin{array}{l}\text { Institute of Theoretical Physics, Academia Sinica } \\
\text { liuc@itp.ac.cn }\end{array}$ \\
\hline 刘显奋 & Liu Yu-fen & Institute of Theoretical Physics, Academia Sinica \\
\hline 刘玉唫 & Liu Yu-xin & $\begin{array}{l}\text { Physics Department, Beijing University } \\
\text { yxliu@itp.ac.cn }\end{array}$ \\
\hline 龙桂鲁 & Long Gui-lu & $\begin{array}{l}\text { Physics Department, Qsinghua University, Beijing } \\
\text { gllong @ mail.tsinghua.edu.cn }\end{array}$ \\
\hline & Moris F. Low & $\begin{array}{l}\text { University of Queensland, Australia } \\
\text { molow @mailbox.uq.edu.qu }\end{array}$ \\
\hline 吕才典 & Lï Cai-dian & $\begin{array}{l}\text { Hiroshima University } \\
\text { lucd@theo.phys.sci.hiroshima-u.ac.jp }\end{array}$ \\
\hline 吕洪凤 & Lü Hong-feng & $\begin{array}{l}\text { Techenical Physics Department, Beijing University } \\
\text { hflv@263.net }\end{array}$ \\
\hline 马建平 & Ma Jian-ping & $\begin{array}{l}\text { Institute of Theoretical Physics, Academia Sinica } \\
\text { majp@itp.ac.cn }\end{array}$ \\
\hline 马中玉 & Ma Zhong-yu & $\begin{array}{l}\text { China Institute of Atomic Energy } \\
\text { mazy12@iris.ciae.ac.cn }\end{array}$ \\
\hline 松尾 泰 & Matsuo Yutaka & $\begin{array}{l}\text { University of Tokyo, Bunkyo-ku } \\
\text { matsuo@hep-th.phys.s.u-tokyo.ac.jp }\end{array}$ \\
\hline 野田二次男 & Hujio Noda & $\begin{array}{l}\text { Ibaraki University } \\
\text { noda@mito.ipc.ibaraki.ac.jp }\end{array}$ \\
\hline 大林惇子 & Atuko Obayashi & \\
\hline 大林治夫 & Haruo Obayashi & $\begin{array}{l}\text { Nagoya University } \\
\text { obay@quartz.ocn.ne.jp }\end{array}$ \\
\hline 小川修三 & Shuzou Ogawa & Nagoya University \\
\hline 大貝義郎 & Yoshio Ohnuki & $\begin{array}{l}\text { Nagoya Women's Univeristy } \\
\text { ohnuki@ @agoya-wu.ac.jp }\end{array}$ \\
\hline 大潼由一 & Yoshikazu Ootaki & $\begin{array}{l}\text { Ibaraki University } \\
\text { nm8105@mcs.ipc.ibaraki.ac.jp }\end{array}$ \\
\hline 大規昭一郎 & Shoichirou Ootsuki & $\begin{array}{l}\text { Kyushu University } \\
\text { otkslscp@ mbox.nc.kyushu-u.ac.jp }\end{array}$ \\
\hline 欧阳钟灿 & Ouyang Zhong-can & $\begin{array}{l}\text { Institute of Theoretical Physics, Academia Sinica } \\
\text { ouyang@itp.ac.cn }\end{array}$ \\
\hline 坂田文彦 & Fumihiko Sakata & $\begin{array}{l}\text { Ibaraki University } \\
\text { sakata@mito.ipc.ibaraki.ac.jp }\end{array}$ \\
\hline 三田一郎 & Ichiro Sanda & $\begin{array}{l}\text { Nagoya University } \\
\text { sada@eken.phys.nagoya-u.ac.jp }\end{array}$ \\
\hline 関 曉之 & Akiyuki Seki & $\begin{array}{l}\text { Ibaraki University } \\
\text { nm9113@mcs.ibaraki.ac.jp }\end{array}$ \\
\hline
\end{tabular}




\begin{tabular}{|c|c|c|}
\hline 单连友 & Shan Lian-you & $\begin{array}{l}\text { Institute of Theoretical Physics, Academia Sinica } \\
\text { shanly@itp.ac.cn }\end{array}$ \\
\hline 沈彩万 & Shen Cai-wan & $\begin{array}{l}\text { Institute of Theoretical Physics, Academia Sinica } \\
\text { cwshen@itp.ac.cn }\end{array}$ \\
\hline 繁田岳美 & Takemi Shigeta & $\begin{array}{l}\text { Ibaraki University } \\
\text { nd7404@mcs.ibaraki.ac.jp }\end{array}$ \\
\hline 宋行长 & Song Xing-chang & Physics Department, Beijing University \\
\hline 菅原祐二 & Yuji Sugawara & $\begin{array}{l}\text { University of Tokyo, Bunkyo-ku } \\
\text { sugawara @ hep-th.phys.s.u-tokyo.ac.jp }\end{array}$ \\
\hline 孙宝坌 & Sun Bao-xi & $\begin{array}{l}\text { Institute of Theoretical Physics, Academia Sinica } \\
\text { sunbx@itp.ac.cn }\end{array}$ \\
\hline 王国利 & Wang Guo-li & $\begin{array}{l}\text { Institute of Theoretical Physics, Academia Sinica } \\
\text { wanggl@itp.ac.cn }\end{array}$ \\
\hline 王 青 & Wang Qing & Physics Department, Qsinghua University, Beijing \\
\hline 吴济民 & $\mathrm{Wu} \mathrm{Ji-min}$ & Institute of High Energy Physics, Academia Sinica \\
\hline 吴 可 & Wu Ke & $\begin{array}{l}\text { Institute of Theoretical Physics, Academia Sinica } \\
\text { wuke@itp.ac.cn }\end{array}$ \\
\hline 吴小红 & Wu Xiao-hong & $\begin{array}{l}\text { Institute of Theoretical Physics, Academia Sinica } \\
\text { wuxh@itp.ac.cn }\end{array}$ \\
\hline 吴锡真 & Wu Xi-zhen & $\begin{array}{l}\text { China Institute of Atomic Energy } \\
\text { lizwux@iris.ciae.ac.cn }\end{array}$ \\
\hline 吴岳良 & Wu Yue-liang & $\begin{array}{l}\text { Institute of Theoretical Physics, Academia Sinica } \\
\text { ylwu@itp.ac.cn }\end{array}$ \\
\hline 熊传胜 & Xiong Chuan-sheng & Physics Department, Beijing University \\
\hline 许甫荣 & Xu Fu-rong & Techenical Physics Department, Beijing University \\
\hline 徐 湛 & Xu Zhan & Physics Department, Qsinghua University, Beijing \\
\hline 许仲平 & Xu Zhong-ping & University of Massachusetts Dartmouth \\
\hline 山上滋 & Shigeru Yamagami & $\begin{array}{l}\text { Ibaraki University } \\
\text { yamagami@ mito.ipc.ibaraki.ac.jp }\end{array}$ \\
\hline 山口直人 & Naoto Yamaguchi & $\begin{array}{l}\text { Ibaraki University } \\
\text { nm9133@mcs.ibaraki.ac.jp }\end{array}$ \\
\hline 晏世伟 & Yan Shi-wei & $\begin{array}{l}\text { Ibaraki University } \\
\text { yansw@mito.ipc.ibaraki.ac.jp }\end{array}$ \\
\hline 杨金民 & Yang Jin-min & Tohoku University \\
\hline 杨立铭 & Yang Li-ming & $\begin{array}{l}\text { Physics Department, Beijing University } \\
\text { lmyang@sun.ihep.ac.cn }\end{array}$ \\
\hline 杨 树 & Yang Shu & $\begin{array}{l}\text { Institute of Theoretical Physics, Academia Sinica } \\
\text { yangs @itp.ac.cn }\end{array}$ \\
\hline 安野 愈 & Masaru Yasuno & $\begin{array}{l}\text { Nagoya University } \\
\text { yasuno@nuc-th.phys.nagoya-u.ac.jp }\end{array}$ \\
\hline
\end{tabular}




\begin{tabular}{|c|c|c|}
\hline 衣学喜 & Yi Xue-xi & Institute of Theoretical Physics, Academia Sinica \\
\hline 米谷民明 & Tamiaki Yoneya & $\begin{array}{l}\text { Univeristy of Tokyo } \\
\text { tam@help1.c.u-tokyo.ac.jp }\end{array}$ \\
\hline 吉田龍生 & Tatsuo Yoshida & $\begin{array}{l}\text { Ibaraki University } \\
\text { yoshidat@mito.ipc.ibaraki.ac.jp }\end{array}$ \\
\hline 喻 明 & Yu ming & Institute of Theoretical Physics, Academia Sinica \\
\hline 虞 跃 & Yu Yue & Institute of Theoretical Physics, Academia Sinica \\
\hline 岳崇兴 & Yue Chong-xing & Henan Normal University, Henan, China \\
\hline 张启仁 & Zhang Qi-ren & $\begin{array}{l}\text { Techenical Physics Department, Beijing University } \\
\text { zhangqr@sun.ihep.ac.cn }\end{array}$ \\
\hline 张肇西 & Zhang Zhao-xi & $\begin{array}{l}\text { Institute of Theoretical Physics, Academia Sinica } \\
\text { zhangzx@itp.ac.cn }\end{array}$ \\
\hline 张宗烨 & Zhang Zhong-ye & Institute of High Energy Physics, Academia Sinica \\
\hline 赵恩广 & Zhao En-guang & $\begin{array}{l}\text { Institute of Theoretical Physics, Academia Sinica } \\
\text { egzhao@itp.ac.cn }\end{array}$ \\
\hline 赵光达 & Zhao Guang-da & Physics Department, Beijing University \\
\hline 赵万云 & Zhao Wan-yun & Institute of Theoretical Physics, Academia Sinica \\
\hline 赵维勤 & Zhao Wei-qin & $\begin{array}{l}\text { Institute of High Energy Physics, Academia Sinica } \\
\text { chaowq@hp.ccast.ac.cn }\end{array}$ \\
\hline 张元仲 & Zhong Yuan-zhong & Institute of Theoretical Physics, Academia Sinica \\
\hline 周先荣 & Zhou Xian-rong & $\begin{array}{l}\text { Institute of Theoretical Physics, Academia Sinica } \\
\text { xrzhou@itp.ac.cn }\end{array}$ \\
\hline 周宇峰 & Zhou Yu-feng & Institute of Theoretical Physics, Academia Sinica \\
\hline 朱传界 & Zhu Chuan-jie & Institute of Theoretical Physics, Academia Sinica \\
\hline 朱守华 & Zhu Shou-hua & Institute of Theoretical Physics, Academia Sinica \\
\hline 朱重远 & Zhu Zhong-yuan & $\begin{array}{l}\text { Institute of Theoretical Physics, Academia Sinica } \\
\text { zzy@itp.ac.cn }\end{array}$ \\
\hline 庄鹏飞 & Zhuang Peng-fei & Physics Department, Qsinghua University, Beijing \\
\hline 卓益忠 & Zhuo Yi-zhong & $\begin{array}{l}\text { China Institute of Atomic Energy } \\
\text { zhuoyz@iris.ciae.ac.cn }\end{array}$ \\
\hline 邹冰松 & Zou Bing-song & Institute of High Energy Physics, Academia Sinica \\
\hline
\end{tabular}




\section{CONTENTS}

Foreword

List of Participants ix

Aspects of Current Particle Physics

M. Kobayashi

CP Violation Past, Present and Future

A. I. Sanda

Probing the Electroweak Symmetry Breaking Mechanism at High Energy Colliders

Y.-P. Kuang

Nonlinear Science in Nuclear Physics

F. Sakata

Review of the Decays of B to $\eta^{\prime} X_{s}, \eta^{\prime} K\left(K^{*}\right)$, and $\eta K\left(K^{*}\right)$

D. $D u$

Physics and Society: The Development of Particle Physics in

Wartime and Postwar Japan

M. Low

String Theory - Where are We Now?

T. Yoneya

From Model to Theory - Progress in Quarkonium Physics $Y .-Q$. Chen

The Measurement Problem in Quantum Mechanics

S. Ogawa

A Relation between Commutative and Noncommutative

Descriptions of D-Branes

N. Ishibashi

The Descent Equation of Noncommutative Differential

Geometry on Lattice

$K . W u$ 
xiv

Tachyon Condensation and Boundary States in Bosonic String Y. Matsuo

Supersymmetry for Flavors

C. Liu

Topological String on $A d S_{3} \times \mathcal{N}$

Y. Sugawara

Boltzmann Transport Equation and $H$ Function in Dilute

Quantum Gas

S. Kanno

Bag Model and the $\pi$-Nucleon Vertex

Q.-R. Zhang

Dominant Gate Imperfection in Grover's Quantum Search Algorithm

G.-L. Long, Y.-S. Li, W.-L. Zhang, and C.-C. Tu

Microscopic Dynamics of Dissipation in Nuclear Collective Motion

S. Yan, F. Sakata, Y. Hashimoto, Y.-Z. Zhuo, and X.-Z. Wu

Possible New Physics Forms for $Z b \bar{b}$ Couplings

R. J. Oakes, J. M. Yang, and B.-L. Young

Quantization on Manifolds and Induced Gauge Potentials

Y. Ohnuki

CP Violations in Charmless Hadronic Two-Body B Decays C.-D. Lü

BRST Cohomological Analysis of Chiral Anomaly and Noncommutative Differential Calculus in Lattice $U(1)$ Gauge Theory

T. Fujiwara

Tensor Categories as a Mathematical Language Describing

Quantum Symmetries

S. Yamagami

Chiral Lagrangian in QCD

Q. Wang and Z.-M. Wang

Contribution of Dirac States on Giant Monopole Resonances 
Cosmic Ray Acceleration in Young Supernova Remnants

T. Yoshida and S. Yanagita

High Spin Superdeformed Bands and the Algebraic Model Description 259 Y.-X. Liu

$\pi$ Nuclear Double Charge Exchange Reaction and Nucleon Correlation 273 Y.-J. Ren, L. Guo, B.-X. Sun, C.-W. Shen, and E.-G. Zhao 